Ueber die Entstehang and Tendenz von Brentanos Gustav Wasa gibt uns folgende Stelle in dem Briefe Dorotheens an Schleiermacher rom 16. Juni 1800 (Ans Schleiermachers Leben III 190) näheren Aufschluss: 'Wir haben hier seit einiger Zeit hübschen Spass mit einigen Bewondrern und Nachahmern von Tieck und Friedrich, die anch in Tiecks Journal tüchtig persiflirt werden. Der eine ist Clemens Brentano; der legt sich darauf Tiecks Nachahmer zn seyn; und schämt sich seiner sentimentalen Ader, die er doch gar nicht verlengnen kann. Er hat eine Farçe geschrieben, "(Instav Wasa", worin er glaubt, der Tieck des Tiecks zu sein; es ist aber herzlich dumm and toll, und klingt doch wie Tieck ungefahr, sodass sich dieser tächtig darüber erbosst, and darum hat er ihn auch so derb mitgenommen im Journal. Uns hat er aber den Anfang eines sentimentalen Romans zu lesen gegeben; der ist nugleich besser, and das verdriesst ihn nan wieder, er will von Teufels Gewalt satyrisch sein. Kurz es ist ein Hauptspass! ${ }^{\star}$ Am 25. Juli 1800 schreibt Dorothea an Brentano (Dorothea I [17]): 'Apropos von Prophezeihnngen. Sie haben eine recht gute auf sich selber im "Wasa" ausgesprochen, nemlich: "Dass Richter nicht mein Gott sei." Nehmen Sie sich in Acht, dass er es nicht doch noch einmal wird!' Im Sommer 1800 muss auch das Bändchen bereits veröffentlicht gewesen sein, denn in dem Briefe vom 29. Juli 1800 an Schiller citiert es Goethe unter den Schriften, welche ihm der Znfall zugeführt babe. 
Brentanos 'Gustav Wasa' schliesst sich unmittelbar und ohne Schlusspunkt an Kotzebues 'Hyperboraischen Esel' an. Ueber die Auffiuhrung des letzteren in Leipzig wăhrend der Herbstmesse 1799 berichtet Caroline an ihre Tochter Auguste (Caroline I 272): 'Kotzebue hat ein Stück gegen die Schlegel gemacht und wahrend der Messe aufführen lassen. Eine Rolle darin ist aus den Fragmenten im Athenkum ausgeschrieben, und soll so den Friedrich vorstellen, der zaletzt ins Tollhaus geschickt wird. Uebrigens platterdings kein Witz darin 2usser der Schlegels ihr eigner. Es hat grossen Larm im Parterr gegeben pro und contra - das pro hat natürlich bey den Leipzigern die Oberhand behalten, hinterher hat Müller*) aber die weitere Auffihrung verbieten lassen. Das Stück heisst der hyperboreische Esel oder die Bildnng nnserer Zeit. Du kannst leicht denken, wie sich Schlegel tout de bon daran ergötzt hat. Es ist Dir ein Tausendspass.'

Noch im Herbste 1799 (die 'Zueignungsschrift' ist vom September 1799 nnterzeichnet) erschien Kotzebue's Parodie im Drack: 'Der hy perboreische Esel oder die heutige Bildung. Ein drastisches Drama, und philosophisches Lustspiel für Jünglinge, in Einem Akt. Von A. v. Kotzebue. Leipzig, bey Paul Gotthelf Kummer, 1799.' Die Vignette auf dem Titelblatte stellt einen vor dem Standbilde des Apoll tanzenden Esel vor, zur Verlöhnnng des Athenăumsfragmentes von A. W. Schlegel, welches lantet (Friedrich Schlegels Jngendschriften II 234): 'Schwerlich hat irgend eine andre Litteratur so viele Ausgebarten der Originalitatssucht aufzuweisen als unsre. Es zeigt sich anch hierin dass wir Hyperboreer sind. Bey den Hyperboreern wurden namlich dem A pollo Esel geopfert, an deren wunderlichen Sprïngen er sich ergötzte.' Unter der Vignette findet sich das Motto aus

*) Der Bitrgermeister von Leipzig: Karl Wilhelın Mitller; vgl. Uber ihn Minor, Christian Felix Weisse S. $17 \mathrm{f}$. 
Virg. Ecl. 5,75: ,Saltantes Satyros imitabitur —' Damit ist anch der Titel der Kotzebneschen Satire erklurt, welche ihr Verfasser in einer eigenen Zueignungsschrift kecklich den 'Herren Verfassern und Herausgebern des Athenăm' widmet: er habe sie nar geschrieben, um ihre Lehren ins grosse Pablikum zu bringen ('Sie wissen ja, ich schreibe nur für den grossen Haufen') and sie folglich gemeinnütziger zu machen. Finem Freunde gegenüber, welcher die Fragmente hasse, habe er sich anheischig gemacht, sie in dramatischer Form darzustellen, dass jeder seine Frende haben solle.

Wir müssen Carolinens Urteil über die Salzlosigkeit dieses Spasses bestatigen. Der ganze Witz besteht in der Citatenrolle Karls, der sich schon ausserlich (mit randgeschnittenem Haar and nachlässiger Kleidung) genan 80 wie der junge Friedrich Schlegel präsentiert (vgl. ans Schleiermachers Leben I 170) and welcher durchans mit den durch gesperrten Drack and genane Ortsangabe heransgehobenen Worten der Gebrüder Schlegel redet. Die Widerlegang geschieht anf dieselbe Weise wie in Nicolais 'Vertrauten Briefen von Adelheid $B^{* *}$ an ihre Freundin Julie $S^{* *}$ ' Karl hat anf der Universitat Jena bei Fichte die Wissenschaftslehre, bei $A$. W. Schlegel Aesthetik, bei Schiller Geschichte gehört and kehrt nun als ein völlig verschrobener, untauglicher Mensch in den Kreis der Seinen zurück. Er stősst zuerst seine Mutter (Fran von Berg) durch einige egoistische Fragmentsatze von sich ab; seinem Oheim nnd zukünftigen Schwiegervater, dem Baron Krenz, erscheint er mit seinen Gedanken vom Genie als ein Narr; seiner Geliebten (Malchen, der Tochter des Barons) trägt er die Moral der Lucinde vor; dem Fürsten, der als deus ex machina anftritt, macht er sich durch seine revolutionären Fragmente verhasst, and darch die aus der Lacinde vorgetragene Lehre vom Miissiggang verdirbt er sich noch daza die Anstellung. Wie Nicolai seinem Gundibert einen nüchternen Genossen an die Seite stellt, der mit 
praktischem Sinne dort erntet, wo Gundiberts falsche Philosophie nar zerstört: so steht anch hier dem herzlosen und verschrobenen Philosophen ein simpler, bescbrankter Bruder (Hans) gegenüber, der sich als Lebensretter auszuzeichnen Gelegenheit erhält nnd schliesslich Stellung and Braut dem Bruder vorwegnimmt. Dieser erscheint in der sittlichen Ereiferung des Dichters als der grösste moralische Vagabund, der nichts von Pflichten gegen den Staat, Gott und Mitbürger weiss, der aber freilich (um anch das weiche Herz zur Geltung kommen zu lassen) eher Mitleid als Zorn verdient. Die ganze hentige Bildnng ist impertinente Anmassung, hochtrabender Unsinn and gänzliche Notzlosigkeit.

Hatte Kotzebue hier weiter nichts gethan, als die Athenăumstragmente und die Moral der Lucinde anf das Eis geführt, so lag es nahe seinen Dramen das gleiche zu thun. Noch Anfangs 1800, in den ersten Tagen des neuen Jahrhunderts, machte ein historisches Schauspiel 'Gustav Wasa' auf dem Weimarischen Theater sein Glück: Kotzebue, der sich allen Richtungen anzuschmiegen und aus allen Anregungen für seine Mase Vorteil zu ziehen verstand, machte sich hier sogleich den Ton und die Iamben des Schillerschen Wallenstein zu Nutze. An diese letzte Erscheinnng hielt sich Brentano, welcher als Racher des 'Hyperboreischen Esels' anftrat, noch ehe A. W. Schlegel seine 'Triumphpforte' begann. Schon der Titel bezeichnet die Parodie als eine Fortsetzung des 'Hyperboreischen Esels': denn der erste Druck des letzteren hat eben 58 Seiten, den Inhalt der 58. Seite and den Schluss des Ganzen bildet das unten S. 11 abgedruckte Stïck, an welches Brentano ohne Schlnsspunkt seine Satire anknüpft. An den letzten Satz des Fürsten bei Kotzebne: 'Was könnte einem Fürsten willkommner sein, als das hänsliche Glück seiner Unterthanen' - reiht Brentano unmittelbar die Einladung, mit ihm nach der Residenz in die von Kotzebue zu dem Vermăhlungsfeste Hansens gedichtete Komödie 'Gustav Wasa' zu falıren. 
Brentano lässt noch einige Familienglieder und die Dienerschaft hinzntreten, welche bei Kotzebue nicht vorkommen, and die ganze Gesellschaft wird mittelst einer 'Wurst' zum Schauspiel abgeführt.

Alle die folgenden Scenen, welche sich mit dem Stück im Stücke beschäftigen, welche die Entstehung und Vorbereitung des 'Gustar Wasa' betreffen oder seine Auffihrung anterbrechen, stehen dentlich unter dem Einflusse von Tiecks satirischen Marchenkomödien. Zunăchst führt uns Brentano in die Weimarische Bibliothek, wo sich Kotzebues Lnstspiele and Schanspiele mit den alten Klassikern und Kirchenvätern herumstreiten, welche mit den Worten ihrer eigenen Schriften redend eingeftihrt werden. Wie der Prince de Conty (wenn anders anter ihm der Verfasser des Paragone della poesia tragica d'Italia con quella di Francia 1732, einer der ersten Bekămpfer der französischen Tragödie, za verstehen ist) in diese Gesellschaft kommt, ist nicht leicht einzusehen. In der Rede des Bibliothekars ist die litterarische Satire am starksten and deatlichsten. Brentano ergreift energisch die Partei der beiden Schlegel. Nach Wilhelms Brach mit der Jenaer Literatarzeitung, wodurch diese den einzigen scharfen Zahn verloren hatte, bilden beide Bräder 'Simsons Kinnbacken fürchterlich.' Die Verheerung, welche sie anrichten, wird mit parodistischer Benutzung der 'Glocke' and mit hauptsłchlicher Verspottung der Schillerschen Reime dargestellt: 'tiber ein Gedicht von Schiller, das Lied von der Glocke', schreibt ja anch Caroline im Oktober 1799 an ihre Tochter (Caroline I 272), 'sind wir gestern Mittag fast von den Stüblen gefallen vor Lachen, es ist a la Voss, a la Tieck, a la Teufel, wenigstens um des Teufels zu werden.' Als das Jahrhundert ganz niedergemacht ist, bleibt nichts bestehen als die Schlegelschen Fragmente. Wie nach dem Gewittersturm die Morgenröte and die Lacinde erscheint, muss Jacobi's von Friedrich Schlegel gerichteter 'Woldemar' erzăhlen und Jakob Böhme antwortet: 'Wen̊ 
zwei Sonnen zngleich anfgehen, so ist ein grosser herrlicher Tag vorhanden, and der Bräntigam ist im Anzug.' Wie hier, so finden wir anch am Schlusse Brentano als Eiferer für die Schlegelsche Sekte: das 'literarische Bedlam Gähna' d. h. das im auf klărerischen Teile Dentschlands als Hochschule der Narrheit verrufene Jena muss nach ihm 'Betlehem' heissen and den Titel der Schlegelschen Lucinde legt er ziemlich gleichbedentend mit Morgenröte als Lax-inde ans, indem er zu dem einen c noch ein zweites fügt.

Die folgenden Gartenscenen, in welchen die Anfknüpfung des Herrn Abonnement die Anfführung des Gustav Wasa bei abonnement suspendu bedenten soll, nennt Brentano selbst einmal ein Seitenstück zam Garten der wahren Poesie in Tiecks Zerbino, ohne dass indessen die Aehnlichkeit über das änsserliche hinansginge. Anspielongen anf $W$ eimarer Oertlichkeiten, wie den gening hnins loci mit dem von einer Schlange nmzingelten Marmorblock (vgl. den zwölften Stahlstich in Diezmanns 'Weimar-Album' 1860) sind ebenso deutlich wie die Ansfalle anf den Satiriker Falk, welchen anch Tieck nicht unangefochten lassen konnte, und den Kammerherrn von Einsiedel. Bei der Verhöhnung von Knigges 'Umgang mit den Menschen' müssen wir an die Polemik denken, welche Schleiermacher diesem Buche zugedacht hatte, and deren Gedanken sicher durch Friedrich Scluegel nach Jena gedrungen waren. Brentano zeigt sich auch hier nur als willfähriger Exekutor, der den Willen seiner Oberen vollzieht.

Viel enger noch schliessen sich die Scenen im Schauspielhause an das Vorbild Tieck, besonders an den gestiefelten Kater an - nur dass Brentanos mutwillige Phantasie nicht bei Tieck stehen bleibt, sondern es recht daranf angelegt hat 'den Tieck des Tieck' zu spielen. So unselbstandig Brentano im Inhalte and in der Form seiner Satire ist, so reich und nnerschöpflich ist er in der Ausfibrang des einzelnen. Wie bei Tieck spielen Schau- 
spieler und Publikum mit - Brentano holt noch eine ganze Reihe allegorischer und symbolischer Gestalten herbei. Der Theatergeist, der Vorhang, der gemeine Menschen-Werkeltag und die feierliche Theaternacht ergreifen das Wort. Logengeister heissen die eintretenden Damen willkommen. Unter den letzteren treten die Dame 'Lesbia', d. h. Amalia von Imhof, deren Gedicht die 'Schwestern von Lesbos' in Schillers Almanach auf 1800 erschienen und im oben citierten Briefe Carolinens 'eben weiter nicht viel als ein Rudel Hexameter' genannt worden war, und die 'wolgezogene Dame' $d$. h. Caroline von Wolzogen, die Schwägerin Schillers, Verfasserin des Romanes 'Agnes von Lilien', besonders hervor. Der 'Legationsrichter' d. h. der Legationsrat Jean Paul Friedrich Richter und der 'Herr' der' (Herder), der Verteidiger der Humanität und Bekämpfer der neueren Philosophie, verraten sich sogleich bei ihrem Auftreten durch den Namen. Dann ergreift das ganze erleuchtete Hans, mit Säulen, Wänden, Arabesken und andern Verzierungen das Wort, bis die Symphonie beginnt, welche der Ouverture und Zwischenaktsmusik in Tiecks 'Zerbino' and der 'verkehrten Welt' nachgebildet ist. Wenn dort die Instrumente sich selbst charakterisieren oder die Sätze der Zwischenaktsmusik sich in Worte auflösen, so personifiziert Brentano die einzelnen Instrumente geradezu: die Violinen erscheinen als Jünglinge; die Flöte als Schäfer vom Himmel, der die Wolken hütet; die Bratschen sind die Oekonomen, Schullehrer und Kalendermacher; das Waldhorn, Tiecks Lieblingsinstrument, erscheint als Eremit; die Hoboe als Phantast; die Klarinetten als Zigeuner und Zigeunerinnen. Die musikalische Bedeutung dieser Personifikationen verliert sich schliesslich ganz, sie agieren wie selbständige Wesen und der Dichter ergreift wieder selber das Wort, indem er auf seine Symphonie weiter keine Rücksicht nimmt. Kein Wunder, dass dieses tolle Gewirre einen Offizier unter den Zuschauern $\mathrm{nm}$ die Besinnung bringt!... In derselben Art gehen die Zwischengespräche der Zu- 
schaner während der Anfführung des 'Gustav Wasa' and die Schlnssgespräche, nachdem sie das Theater ans Langeweile verlassen haben, fort. Es treten zwei Transzendentalphilosophen hinza, 'welche noch nicht hinübergekommen sind', und der Rektor Excerpino, der im 'gestiefelten Kater' seiner 'Entwicklung des Ifflandischen Gastspiels' wegen gegeisselte Böttiger, der eine Antipathie vor den Katern hat und anch hier von demselben darch das ganze Stück verfolgt wird. Der 'Stadtrichter', der englisch Hnmorbier ansschenken lässt, ist natürlich wieder Jean Paul. Die Erlanger Litteraturzeitang warde zur Zeit, als Brentanos 'Gustav Wasa' erschien, noch allein von Mensel redigirt: als später Mehmel als Mitredacteur hinzutrat und bei den Genossen des romantischen Kreises am Mitarbeiter warb, fanden wenigstens die philosophischen Artikel in Jena eine günstigere Beurteilung. Das 'Motto zu einer Theorie des Bildungstriebes', welches aus dem Archiv für moralische und religiöse Bildung des weiblichen Geschlechts empfohlen wird, ist für Hülsen ansgewăhlt, dessen Anfsatz 'über den Bildungstrieb' 1800 im Fichte-Niethammerschen Journal erschienen war.

Was nun den Kern des Ganzen, die Parodie des Kotzebueschen 'Gustav Wasa' betrifft, so darf sich dieser freilich nicht im entferntesten mit A. W. Schlegels Triumphpforte vergleichen. Das Element von Nachdichtang, welches jeder Parodie zu Grande liegt, war recht eigentlich A. W. Schlegels Domäne. Er parodiert Kotzebue, indem er ihn und seine Gestalten eben nur so reden lăsst, wie sie reden. Eine solche Parodie setzt eine weniger originelle, aber auch eine reifere Begabung als die Brentanos voraus. Brentano aber hält nicht still : er schüttelt Kotzebue ab, noch ehe er ihn recht aufgeladen hat. Er muss den Kotzebue des Kotzebue hinmalen, um ihn dem Gelächter preis zu geben. Hält man, wie es wol nötig ist, um die Parodie ganz zul geniessen, den Kotzebueschen Text daneben; so findet 
man, dass Brentano denselben noch einmal in's Kotzebuesche ïbersetzl und auch dort, wo sich der Antor davon fermgehalten, die gewohnte Schablone desselben zar Anwendung bringt. Brentano travestiert den Inhalt des 'Gustav Wasa'; indem er den Gang der Scenen im ganzen beibehalt, schreibt er den iambischen Dialog in eine Art zahmer Knittelverse um. Manches konnte er fast wörtlich beibehalten, wie die Stellen, wo Hans dem Wasa das Blut des Vaters auf seinem Aermel zeigt and ihm das von demselben getränkte Tuch überreicht: um dem Leser selber die Vergleichnng zn ermöglichen, lasse ich den Originaltext dieser Scene im Anhange (S. 132 ff.) abdrucken. An anderen Stellen folgt Brentano dem Texte genau, den er aber parodiert; und oft wieder dichtet er, wie gesagt, Kotzebnesche Effekte in satirischer Absicht in Kotzebue hinein. Requisiten, welche Kotzebue zar Anwendung bringt, wie das Spinnrad, die Lampe a. 8. w. ergreifen wiederholt das Wort, und der Kater, welcher sich aus der Tieckischen Märchenkomödie hier eingeschlichen hat und mit den Zuschanern bekannter ist als mit den Personen des 'Gustav Wasa', wird je lknger je mehr zur Hauptrolle. Schon im zweiten Akt beschrankt sich die Parodie auf einzelne hervorragende Momente des Kotzebneschen Drama; im dritten kommt es gar nicht mehr dazu, weil sich das gelangweilte Publikum zurückzieht. Wenigstens hat es Brentano dahin gebracht, eben so viele Personen in seiner Parodie in Bewegang zu setzen als Kotzebue: das Personenverzeichnis des Kotzebneschen 'Gustav Wasa' weist 37 Personen anf, und genau so viele hat sich Brentano zil verschaffen gewnsst; daher die Bemerkung am Schlusse des Personenverzeichnisses.

Die obigen Bemerknngen dürften wohl genügen, um den Leser zam Verständnis der satirischen Bezüge anzuleiten. Im übrigen wird man sich leicht zurecht finden, wenn man auf die Wort- und Namenspiele, in denen Brentano fast immer seine satirischen Absichten verrät, 
einige Aufmerksamkeit verwendet. Die Vorliebe für das Wortspiel wurde durch den Kapuziner in Wallensteins Lager bei den Romantikern angeregt nnd später von A. W. Schlegel in den Berliner Vorlesungen and von Bernhardi in seiner Sprachlehre grundsătzlich gehegt. Brentano hat wie Tieck diese Vorliebe zeitlebens beibehalten und manche seiner frühesten Witzworte, wie z. B. das 'Manlhenkolische' für das Melancholische, fuihrte auch Dorothea gerne im Munde. Im 'Gustav Wasa' wird der Witz fast nur aus diesem Säckel bestritten. Ueber so anffallige Dinge wie jambes and Iamben, Niedt und Hammer (Niethammer), Im Hof (für Imhof), Schütze (Schütz, Herausgeber der Jenaer Litteratarzeitnng) and Hufe Land (der Jurist Hufeland, sein Kollege), sowie über die absichtlichen Druckfehler wie Herder (statt härter), Gering (statt Gerning; vgl. über diesen z. B. Schillers Briefwechsel mit Cotta 452) wird man nicht leicht hinweglesen; aber auch die Naturphilosophen und Mediziner: Ritter, Brown, der Weimarische Leibmedicns Starke a. A. dürfen in der Anweisung. welche Gustav Wasa S. 83 seinem Diener gibt, nicht übersehen werden. Der in der Bibliothekscene auftretende Gren ist der Professor der Chemie an der Universitat Halle und Verfasser anch von Goethe geschatzter naturwissenschaftlicher Werke.

Der folgende Abdruck des Gustav Wasa ist getreu nach dem ersten Drucke, welcher im Jahre 1800 bei Wilhelm Rein, einem Buchhändler in Leipzig erschien, in dessen Verlage ein Jahr vorher eine gegen dic Romantik Partei ergreifende und (irrthümlich) von vielen gleichfalls dem Brentano zugeschriebene Satire 'Die: Diogeneslaterne' ausgegeben worden war. Anch das Druckfehlerverzeichnis des ersten Druckes wurde beibehalten: weil auch in ihm Brentano sein mutwilliges Spiel treibt and weil die Rabriken verkelirt gelesen zagleich die Abweichangen dieses Neudruckes von dem ersten Drucke anzeigen können; denn natürlich habe ich die nnabsicht- 
lichen Druckfehler in dem Neudrucke verbessert, während zu den absichtlichen die Berichtigungen des Druckfehlerverzeichnisses in Klammern hinzugefïgt wurden. Brentano schreibt statt des Namens Gren (s. oben) regelmässig Green; statt der bei Kotzebue vorkommenden Ort - und Personennamen Calmar und Gregerson, ohne dass ein besonderer Grund erkennbar wäre, stets Collmar und Gegerson: ich habe Kotzebue's Schreibung beibehalten. Sonst habe ich den Text an folgenden Stellen geändert :

S. 3 Z. 1 salza del libro aus salza de libro S. 5 Z. 1 Green aus Geen | S. 16 Z. 31 Prince be Conty aus Prince bi Conty | S. $23 \mathrm{Z} .30$ jeben in unt=

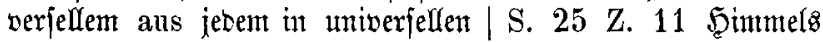
Bolfen aus Simmelsiwolfen | S. 29 Z. 32 einem aus einen | S. 34 Z. 16 Wiffenidaftelere aus Wiffenfduafteere|S. 37 Z. 12 mit foldiem Broo ans foldien|S. 43 Z. 6 Dem aus ben | S. 59 Z. 8 nehmt aus nehm | S. 66 Z. 13 Eein aus Sei | S. 83 Z. 16 Daj aus bas idh nidyt heul |S. 87 Z. 6 Bom aus von jumesifyen Shutgetit | S. 87 Z. 2 Un aus unb ઉdinedens Seeil ein Menthenthein / S. 91 Z. 15 Unt barum aus barum | S. 92 Z. 12 gejdiefin aus ge= farefien |S. 101 Z. 30 barein aus barm |S. 105 Z. 32 Reben aus Meben | S. 107 Z. 30 Hnt aus nun nats bem Razenjdufter | S. 108 Z. 10 joll'n ans jollen | S. 114 Z. 26 meinem aus meinen|S. 115 Z. 4 Precepteur ans

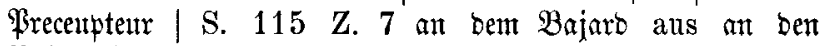
Bajard | S. 117 Z. 1 ifnt wie anf ben \&eib gegoffent aus if)n w. a. b. \&. g. | S. 117 Z. 9 feinem aus feinen | S. 125 Z. 18 unteigentitziger ans eigentitziger |S. 131 Z. 5 aublaften aus erhalten.

Das Citat aus Minutius Felix S. 16 Z. 27, welches im Original fehlerhaft mit Encrius hystrio beginnt, hat der Herausgeber dieser Sammlung richtig gestellt. 
Der Neffe des Dichters, Herr Professor Dr. Franz Brentano in Wien, hat mir das diesem Neudrucke zu Grunde liegende Exemplar des Originaldruckes zweimal zar Verfügung gestellt, wofür ich ihm an dieser Stelle anfrichtigen Dank sage.

Prag, 30. April 1883.

\section{J. Minor.}




\title{
Satiren
}

\author{
und \\ poetifbe Epiele \\ bon \\ Alaria.
}

Erîtez̊ Bänb๘en

Buftapsafa.

\section{Ieipzig, 1800.}

bei $\mathfrak{B}$ ilhelm Rein. 

Da bie Ytaliäner eine Borrebe la salza del libro nennen, fo wollte id leine maden, um bem Bude etrabs ibbrig zu Iaffen; benn bätte id eine folde Botrebe gemadt, fo wirrbe mix gemiß mander mit Redt vorwerfen tönnen, bie ganze

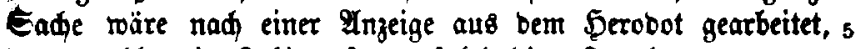

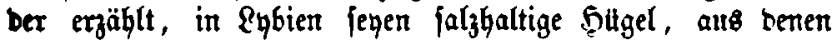
fubc Duellen entipringen. Lebrigens

Un anteur a genoux dans une humble Prêface, An lecteur qu'il ennuye a beau demander grace; n ne gagnera rien sur ce Juge irrité, Qui lai fait son prozes de plaine sutorité.

Mr. DESPREAUX. 